\title{
Liver Grafts for Transplantation from Donors with Diabetes: An Analysis of the Scientific Registry of Transplant Recipients Database
}

\author{
Jun Zheng ${ }^{1,2}$, Jie Xiang ${ }^{1}$, Jie Zhou ${ }^{1}$, Zhiwei $\mathrm{Li}^{1}$, Zhenhua $\mathrm{Hu}^{1}$, Chung Mau Lo ${ }^{2 *}$, Weilin Wang ${ }^{1 *}$ \\ 1 Division of Hepatobiliary and Pancreatic Surgery, Department of Surgery, First Affiliated Hospital, School of Medicine, Zhejiang University, Hangzhou, Zhejiang, China, \\ 2 Department of Surgery, The University of Hong Kong, Hong Kong; 3Key Laboratory of Combined Multi-organ Transplantation, Ministry of Public Health Key Laboratory \\ of Organ Transplantation, Hangzhou, Zhejiang, China
}

\begin{abstract}
Patients with a history of diabetes mellitus (DM) have worse survival than those without DM after liver transplantation. However, the effect of liver grafts from DM donors on the post-transplantation survival of recipients is unclear. Using the Scientific Registry of Transplant Recipients database (2004-2008), 25,413 patients were assessed. Among them, 2,469 recipients received grafts from donors with DM. The demographics and outcome of patients were assessed. Patient survival was assessed using Kaplan-Meier methodology and Cox regression analyses. Recipients from DM donors experienced worse graft survival than recipients from non-DM donors (one-year survival: $81 \%$ versus $85 \%$, and five-year survival: $67 \%$ versus $74 \%, P<0.001$, respectively). Graft survival was significantly lower for recipients from DM donors with DM duration $>5$ years $(P<0.001)$ compared with those with DM duration $<5$ years. Cox regression analyses showed that DM donors were independently associated with worse graft survival (hazard ratio, 1.11; 95\% confidence interval, 1.02-1.19). The effect of DM donors was more pronounced on certain underlying liver diseases of recipients. Increases in the risk of graft loss were noted among recipients from DM donors with hepatitis-C virus (HCV) infection, whereas those without HCV experienced similar outcomes compared with recipients from non-DM donors. These data suggest that recipients from DM donors experience significantly worse patient survival after liver transplantation. However, in patients without HCV infection, using DM donors was not independently associated with worse post-transplantation graft survival. Matching these DM donors to recipients without HCV may be safe.
\end{abstract}

Citation: Zheng J, Xiang J, Zhou J, Li Z, Hu Z, et al. (2014) Liver Grafts for Transplantation from Donors with Diabetes: An Analysis of the Scientific Registry of Transplant Recipients Database. PLoS ONE 9(5): e98104. doi:10.1371/journal.pone.0098104

Editor: Michael Zimmerman, University of Colorado School of Medicine, United States of America

Received December 30, 2013; Accepted April 28, 2014; Published May 21, 2014

Copyright: $\odot 2014$ Zheng et al. This is an open-access article distributed under the terms of the Creative Commons Attribution License, which permits unrestricted use, distribution, and reproduction in any medium, provided the original author and source are credited.

Funding: This work was supported by grants from the Major State Basic Research Development Program of China (973 Program) (No. 491010-N51308) and National Natural Science Foundation of China (No. 491010-N11180). The funders had no role in study design, data collection and analysis, decision to publish, or preparation of the manuscript.

Competing Interests: The authors have declared that no competing interests exist.

*E-mail: zywangw@@163.com (WLW); chungmlo@hku.hk (CML)

\section{Introduction}

The growing incidence of type-2 diabetes mellitus (DM) worldwide is recognized as one of the greatest challenges to public health [1]. In 2011, 366 million people worldwide had DM, and this figure is expected to rise to 552 million by 2030 [2]. In general, DM patients suffer worse health-related outcomes than non-DM patients in many medical conditions, and this is also true among liver transplantation (LT) patients [3].

LT is a well-recognized treatment for patients with end-stage liver disease (ESLD) and/or hepatocellular carcinoma (HCG). Ten-year survival of 59\% and $83 \%$ for deceased donor and living donor transplantation, respectively, has been recorded [4]. In recipients, the role of $\mathrm{DM}$ as an independent risk factor for poor survival after LT has been examined explicitly in several studies: a higher mortality in DM patients than in non-DM patients has been observed [3,5-7]. The use of post-transplantation immunosuppressive drugs and other DM-associated factors (e.g., poor wound healing, impaired neutrophil function, obesity, microvascular/macrovascular disease) may result in poor outcomes among DM patients undergoing LT [8]. However, whether or not DM donors have a negative influence on the outcomes of LT recipients is not known. Only a few studies regarding the effect of DM donors on survival have been carried out. Thus, the goal of the present study was to determine if DM donors affected the mortality of LT patients after the procedure.

Based on a national registry database in the USA, we assessed the LT outcomes of patients who received DM donor grafts and compared the results with those who received non-DM donor grafts. We wanted to know whether we could use DM donors safely for patients with ESLD.

\section{Patients and Methods}

\section{Ethical Statement}

The study protocol was approved by the Research Ethical Committee of Zhejiang University (Zhejiang, China). Written informed consent was obtained from all participants. The patient records/information was anonymized and de-identified prior to analysis in the database. 


\section{Data sources}

This study used data from the Scientific Registry of Transplant Recipients (SRTR). The SRTR data system includes data on all donor, wait-listed candidates, and transplant recipients in the US, submitted by the members of the Organ Procurement and Transplantation Network (OPTN), and has been described elsewhere. The Health Resources and Services Administration (HRSA), US Department of Health and Human Services provides oversight to the activities of the OPTN and SRTR contractors [9].

\section{Study cohort}

All LT patients who received a first isolated LT between 1 January 2004 and 31 December 2008 were eligible for inclusion into the study. Donors were considered to have DM if positive responses to the variable "donor's history of diabetes" or "donor's duration of diabetes" were recorded. Donors were considered to be non-diabetic if negative responses were recorded for these variables. Recipients for whom a donor history of DM was not known were excluded from the study.

Potentially confounding factors for donors and recipients were examined. Recipient characteristics were: age; sex; ethnicity; history of DM; history of HCC; whether the patients was receiving artificial ventilation; whether the patient was undergoing dialysis in the week before orthotopic liver transplantation (OLT); model for end-stage liver disease (MELD) score; serum levels of creatinine; serum level of bilirubin; and cause of liver disease.

Causes of liver disease were categorized as: hepatitis B virus (HBV); hepatitis $\mathrm{C}$ virus (HCV); alcohol; non-alcoholic steatohepatitis (NASH); autoimmune disease (autoimmune hepatitis, primary biliary cirrhosis, primary sclerosing cholangitis); and other causes. Patients classified with HCV in addition to another diagnosis were included under a diagnosis of HCV. Patients who had a diagnosis of HCC were included in the cohort under their primary cause of liver disease.

Donor variables were: age; sex; ethnicity; history of hypertension; body mass index (BMI); donor risk index (DRI) [10]; donation after cardiac death (DCD) donor; warm ischemia time (WIT); cold ischemia time (CIT); and cause of death.

\section{Outcome measures}

The main outcomes were patient survival and graft function. Current status and time-to-outcome were included as outcome measures. Patient follow-up was defined as the time from transplantation until the date of death or last known follow-up. The occurrence and date of death were obtained from data reported by the transplantation centers, and were completed using data from the US Social Security Administration and OPTN.

\section{Statistical analyses}

The study cohort was compared for baseline characteristics with regard to recipients and donors. Statistical analyses were carried out using the Student's $t$-test for continuous variables, and the chisquare test for categorical variables. Survival was assessed using Kaplan-Meier curves and compared with log-rank tests. Cox proportional hazard models were created for the time-to-survival and time-to-graft loss to evaluate the potential predictors of the outcome measures. Variables that were significantly different in the baseline comparison as well as those clinically relevant even if similar at baseline were included in the models. Results were expressed as hazard ratios (HRs) with 95\% confidence intervals (CIs). The causes of graft loss and patient death were analyzed and compared between cases and controls. Results are the mean \pm standard deviation (SD) unless indicated otherwise. A standard alpha level of 0.05 was taken to indicate statistical significance. All statistical tests were two-sided. Analyses were conducted using SPSS ver15.0 (SPSS, Chicago, IL, USA).

\section{Results}

Out of the 26,645 patients who underwent a first isolated LT during the study period, 25,413 met the inclusion criteria after the exclusion of LTs from donors whose DM histories were not known $(\mathrm{n}=1232,4.6 \%)$. Of these, $2469(9.7 \%)$ had DM and 22,944 $(90.3 \%)$ did not. For the cohorts of recipients with DM donors, the mean duration of follow-up was 32 months. The mean duration of follow-up of recipients with non-DM donors was 34 months.

\section{Baseline characteristics}

The baseline characteristics of recipients from DM donors $(\mathrm{n}=2469)$ and recipients from non-DM donors $(\mathrm{n}=22944)$ are listed in Table 1. DM-donor recipients were older (DM donor $54.0 \pm 9.6$ years vs. non-DM donor $53.0 \pm 10.0$ years; $P<0.001)$ but they (in general) displayed fewer risk characteristics compared with non-DM-donor recipients. They had lower MELD scores (DM donor $20 \pm 9$ vs. non-DM donor $21 \pm 9 ; P<0.001$ ), were less likely to be on artificial ventilation (DM donor $3.7 \%$ vs. non-DM donor $5.0 \% ; P=0.004)$ and were less likely to be on dialysis 1 week before LT (DM donor 5.8\% vs. non-DM donor 8.6\%; $P<0.001$ ). There was, however, a greater proportion of HCG recipients in the DM donor cohort (DM donor 25.6\% vs. non-DM donor $23.6 \% ; P=0.026)$, whereas the proportion of recipients with DM donors was similar (DM donor $23.8 \%$ vs. non-DM donor $22.3 \%$; $P=0.098$ ). In addition, DM donors were associated with more adverse factors of graft quality, such as donor age (DM donor $54.5 \pm 12.9$ years $v s$. non-DM donor $40.2 \pm 17.2$ years; $P<0.001)$, history of hypertension (DM donor $78.3 \%$ vs. non-DM donor $28.2 \% ; P<0.001)$, BMI (DM donor $30 \pm 7$ years $v s$. non-DM donor $26 \pm 6$ years; $P<0.001$ ), DRI (DM donor $2.03 \pm 0.42$ vs. non-DM donor 1.79 $\pm 0.42 ; P<0.001)$, and CIT (DM donor $7.6 \pm 3.6 \mathrm{~h} v s$. non-DM donor $7.4 \pm 3.5 \mathrm{~h} ; P<0.001)$. The mean WIT for DM donor transplantation was $42.5 \pm 18.8 \mathrm{~min}$, while that of non-DM donors was $41.6 \pm 18.9 \mathrm{~min}(P=0.083)$. However, DM donor transplantation was associated with a lower prevalence of nonheart-beating donation (NHBD) (DM donor 3.7\% vs. non-DM donor $5.0 \% ; P=0.003)$.

\section{Overall graft survival}

A total of $859(34.8 \%)$ recipients from DM donors and 6,382 $(27.8 \%)$ recipients from non-DM donors lost their grafts. At 1, 5 and 10 years, graft survival was $85 \%, 74 \%$ and $65 \%$, respectively, for recipients from non-DM donors, and $81 \%, 67 \%$ and $56 \%$, respectively, for recipients from DM donors (log rank $P<0.001$ ) (Fig. 1A).

Data for the duration of DM were available for 2,199 of the $2,469(89 \%)$ donors for whom DM history was known, of whom $1,251(50.7 \%)$ were reported to have durations of $\mathrm{DM}>5$ years. Univariate analyses showed the lowest survival in this category $(\log$ rank $P<0.001$ ) (Fig. 1B).

\section{Predictors of graft loss at multivariate analyses}

Initially, all variables were included in the multivariate analyses, which comprised recipient characteristics (age, sex, ethnicity, cause of liver disease, MELD score, HCC, being on artificial ventilation at the time of LT, dialysis 1 week before LT); and donor characteristics (age, DRI, DCD, history of hypertension).

Table 2 shows the factors identified as significant predictors of graft loss in the entire study cohort using a Cox regression hazard 
Table 1. Comparison of patients who underwent transplantation using liver grafts from diabetes mellitus (DM) donors and those who underwent transplantation using liver grafts from non-DM donors with respect to the baseline characteristics of recipients and donors.

\begin{tabular}{|c|c|c|c|}
\hline Recipient characteristic & DM donors $(n=2469)$ & Non-DM donors $(n=22944)$ & $\mathbf{P}$ \\
\hline Age (years) & $54.0 \pm 9.6$ & $53.0 \pm 10.0$ & $<0.001$ \\
\hline Male & $1725(69.9)$ & $15,487(67.5)$ & 0.018 \\
\hline \multicolumn{4}{|l|}{ Ethnicity } \\
\hline White & $1799(72.9)$ & $16,569(72.2)$ & 0.507 \\
\hline Black & $236(9.6)$ & $2084(9.1)$ & 0.441 \\
\hline Asian & $112(4.5)$ & $1086(4.7)$ & 0.686 \\
\hline Hispanic & $298(12.1)$ & $2987(13.0)$ & 0.186 \\
\hline Other & $24(0.9)$ & $218(1.0)$ & 0.925 \\
\hline DM & $587(23.8)$ & $5118(22.3)$ & 0.098 \\
\hline $\mathrm{HCC}$ & $633(25.6)$ & $5418(23.6)$ & 0.026 \\
\hline \multicolumn{4}{|l|}{ Cause of liver disease } \\
\hline $\mathrm{HCV}$ & $1119(45.3)$ & $10,459(45.6)$ & 0.815 \\
\hline HBV & $120(4.9)$ & $1157(5.0)$ & 0.730 \\
\hline NASH & $152(6.2)$ & $1237(5.4)$ & 0.111 \\
\hline Alcohol & $391(15.8)$ & $3255(14.2)$ & 0.027 \\
\hline Autoimmune disease & $248(10.0)$ & $2465(10.7)$ & 0.304 \\
\hline Other & $439(17.8)$ & $4371(19.1)$ & 0.131 \\
\hline Serum creatinine $(\mathrm{mg} / \mathrm{dL})$ & $1.43 \pm 1.21$ & $1.54 \pm 1.35$ & $<0.001$ \\
\hline Serum bilirubin (mg/dL) & $7.06 \pm 9.78$ & $7.77 \pm 10.27$ & $<0.001$ \\
\hline On artificial ventilation & $92(3.7)$ & $1154(5.0)$ & 0.004 \\
\hline Dialysis within 1 week & $142(5.8)$ & $1962(8.6)$ & $<0.001$ \\
\hline MELD score & $20 \pm 9$ & $21 \pm 9$ & $<0.001$ \\
\hline Donor characteristic & DM donors $(n=2469)$ & Non-DM donors $(n=22,944)$ & $\mathbf{P}$ \\
\hline Age (years) & $54.5 \pm 12.9$ & $40.2 \pm 17.2$ & $<0.001$ \\
\hline Male & $1375(55.7)$ & $13,810(60.2)$ & $<0.001$ \\
\hline \multicolumn{4}{|l|}{ Ethnicity } \\
\hline White & $1457(59.0)$ & $15,852(69.1)$ & $<0.001$ \\
\hline Black & $558(22.6)$ & $3433(15.0)$ & $<0.001$ \\
\hline Asian & $72(2.9)$ & $490(2.1)$ & 0.012 \\
\hline Hispanic & $361(14.6)$ & $2964(12.9)$ & 0.018 \\
\hline Other & $21(0.8)$ & $205(0.9)$ & 0.899 \\
\hline BMI $\left(\mathrm{kg} / \mathrm{m}^{2}\right)$ & $30 \pm 7$ & $26 \pm 6$ & $<0.001$ \\
\hline Hypertension & 1933(78.3) & $6478(28.2)$ & $<0.001$ \\
\hline \multicolumn{4}{|l|}{ Cause of death } \\
\hline Anoxia & $523(21.1)$ & $3338(14.5)$ & $<0.001$ \\
\hline Cerebrovascular accident & $1573(63.7)$ & $9460(41.2)$ & $<0.001$ \\
\hline Head trauma & $324(13.1)$ & $9559(41.7)$ & $<0.001$ \\
\hline Other & $49(2.1)$ & $587(2.6)$ & 0.093 \\
\hline NHBD & $91(3.7)$ & $1156(5.0)$ & 0.003 \\
\hline DRI & $2.03 \pm 0.42$ & $1.79 \pm 0.42$ & $<0.001$ \\
\hline WIT (min) & $42.5 \pm 18.8$ & $41.6 \pm 18.9$ & 0.083 \\
\hline CIT (h) & $7.6 \pm 3.6$ & $7.4 \pm 3.5$ & $<0.001$ \\
\hline
\end{tabular}

DM: diabetes mellitus; HCC: hepatocellular carcinoma; HCV: hepatitis C virus; HBV: hepatitis B virus; NASH: non-alcoholic steatohepatitis; BMI: body mass index; NHBD: non-heart-beating donor; DRI: donor risk index; WIT: warm ischemia time; CIT: cold ischemia time.

doi:10.1371/journal.pone.0098104.t001

model. DM donors were associated with an increased risk of mortality for patients after LT (HR $=1.11 ; 95 \% \mathrm{CI}=1.02-1.19)$. This increased risk of death for recipients from DM donors was comparable in magnitude to other well-known independent risk predictors of graft loss such as MELD at LT (HR = 1.01 per unit), recipient with $\mathrm{HCC}(\mathrm{HR}=1.34)$, and $\mathrm{DRI}(\mathrm{HR}=1.41$ per unit). 

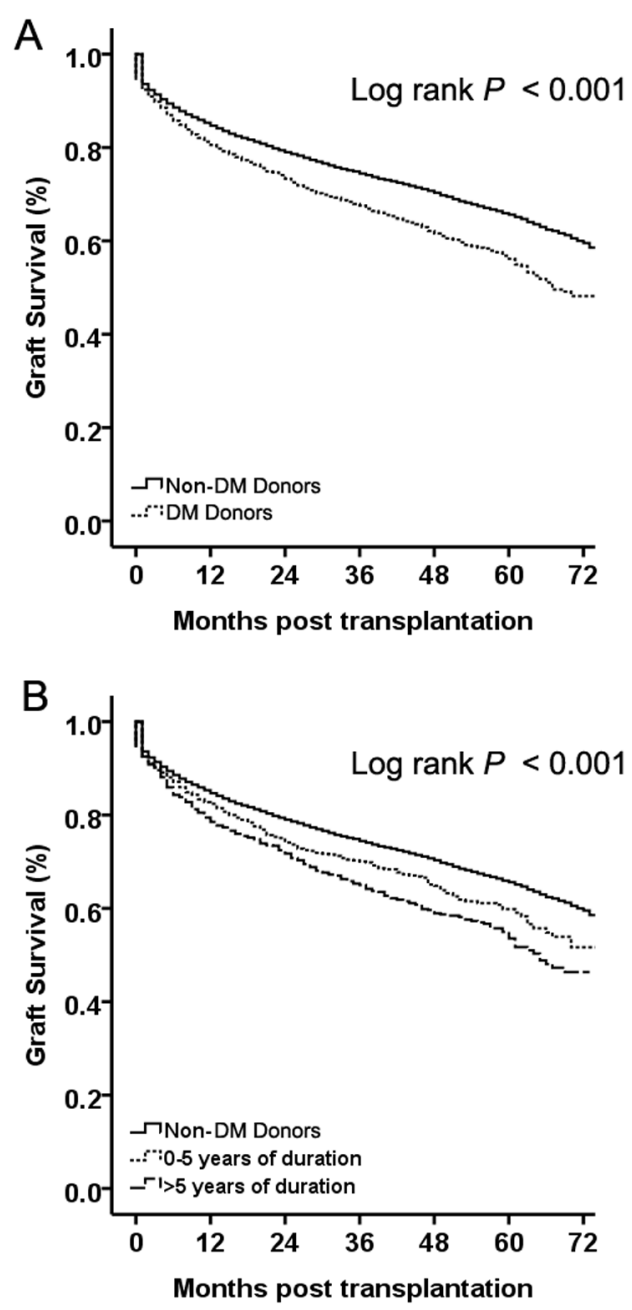

Figure 1. Kaplan-Meier survival curves comparing overall graft survival and graft survival stratified by donor duration of diabetes mellitus (DM) history in recipients from DM and nonDM donors. A) Overall graft survival in recipients from DM and nonDM donors. Recipients with DM donors had significantly lower survival. (B) Graft survival by the duration of DM in the donor. Recipients of liver grafts from DM donors with durations of DM $>5$ years had significantly lower survival compared with those with durations of $\mathrm{DM}<5$ years and recipients from non-DM donors. Continuous line depicts recipients from DM donors and dashed line depicts recipients from non-DM donors. doi:10.1371/journal.pone.0098104.g001
These data showed that a liver graft donated from a subject with DM was an independent risk predictor of graft loss.

\section{Graft survival as related to allocation of DM donors}

A liver graft donated from a subject with DM was an independent risk predictor of graft loss, so we explored the effect of $\mathrm{DM} /$ non-DM donors on graft survival in relation to the underlying disease of recipients (Table 1). Graft survival from DM donors was worse in recipients with $\mathrm{HCV}$ infection, alcoholic liver disease and other liver diseases (Fig. 2A, $P<0.001$; and Fig. 2C, $P=0.014$; respectively). However, the differences between DM and non-DM donors on graft survival disappeared in recipients with $\mathrm{HBV}$ infection, NASH and autoimmune liver disease (Fig. 2B, $P=0.613$; Fig. 2D, $P=0.742$; Fig. $2 \mathrm{E}, P=0.060$; and Fig. $2 \mathrm{~F}$, $P=0.074$; respectively).

The impact of receiving a liver graft from a subject with DM on graft survival in relation to the underlying disease was investigated further in multivariate analyses. To better understand the effects of DM donors on the various diagnoses studied, we plotted the HR (95\% CIs) found in the groups. Only in recipients with HCV was donation from a DM patient an independent predictor of graft loss (Fig. 3).

\section{Discussion}

Using comprehensive clinical data from the SRTR database, the results of the present study suggested that receiving a liver graft from a subject with DM was associated with an increased prevalence of graft loss in a cohort of patients undergoing LT. This increased risk of death remained significant after adjustment for factors for donors and recipients, including those that might represent a selection bias, such as the prevalence of HCC and HCV. Moreover, the present study showed that survival differences were primarily because of lower survival in patients who received liver grafts from donors with a longer duration of DM. If we were to assume that longer duration of DM is a surrogate marker of DM severity, our findings suggest that LT patients who receive liver grafts from subjects with more severe DM may have poorer outcomes. This notion suggests that donors with DM should be employed with caution. This is the first study to compare the outcome of LT recipients from DM donors and nonDM donors.

Even though recipients from DM donors experienced significantly worse graft survival after transplantation compared with those of non-DM donors, a favorable outcome could be expected if a DM donor is transplanted in a favorable condition. Increased risks associated with transplantation for recipients with $\mathrm{HCV}$, HCG or high MELD score have been accepted to extend transplantation to greater numbers of patients in need. We demonstrated that the increased risk of graft loss associated with

Table 2. Cox proportional hazard regression analyses to assess predictors of survival of grafts and patients.

\begin{tabular}{|c|c|c|c|c|}
\hline \multirow[t]{2}{*}{ Variable } & \multicolumn{2}{|c|}{ Univariate } & \multicolumn{2}{|c|}{ Multivariate } \\
\hline & HR & $95 \% \mathrm{Cl}$ & HR & $95 \% \mathrm{Cl}$ \\
\hline DM donors (ref: non-DM donors) & 1.24 & $1.18-1.36$ & 1.11 & $1.02-1.19$ \\
\hline MELD score & 1.01 & $1.01-1.01$ & 1.01 & $1.01-1.02$ \\
\hline HCC (ref: no HCC) & 1.15 & $1.10-1.21$ & 1.34 & $1.25-1.44$ \\
\hline DRI & 1.70 & $1.61-1.79$ & 1.44 & $1.33-1.57$ \\
\hline
\end{tabular}

DM: diabetes mellitus; MELD: Model for End-Stage Liver Disease; HCC: hepatocellular carcinoma; DRI: donor risk index.

doi:10.1371/journal.pone.0098104.t002 
A
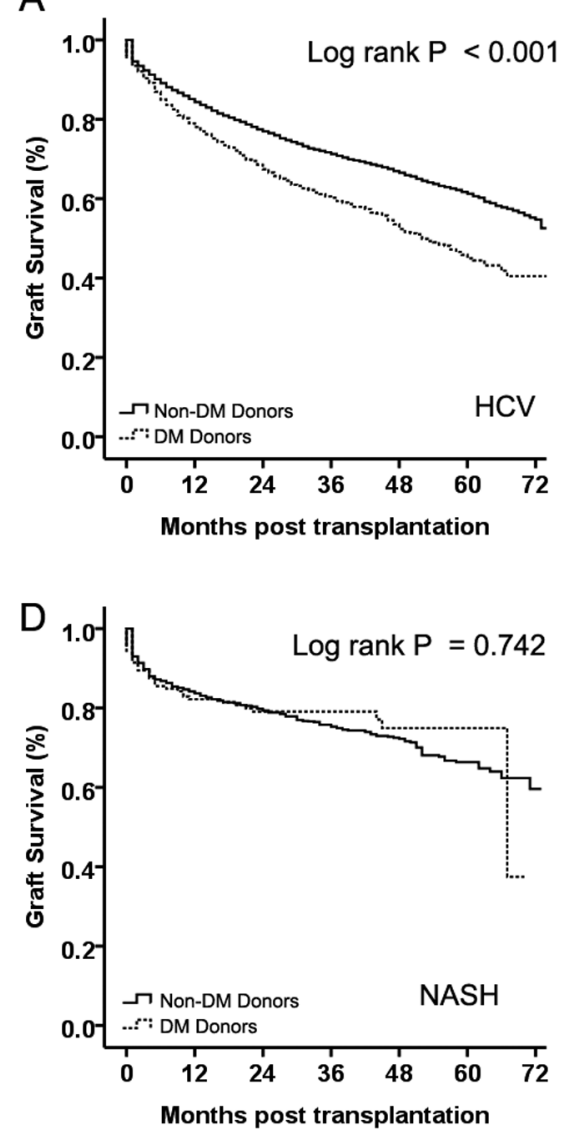

B
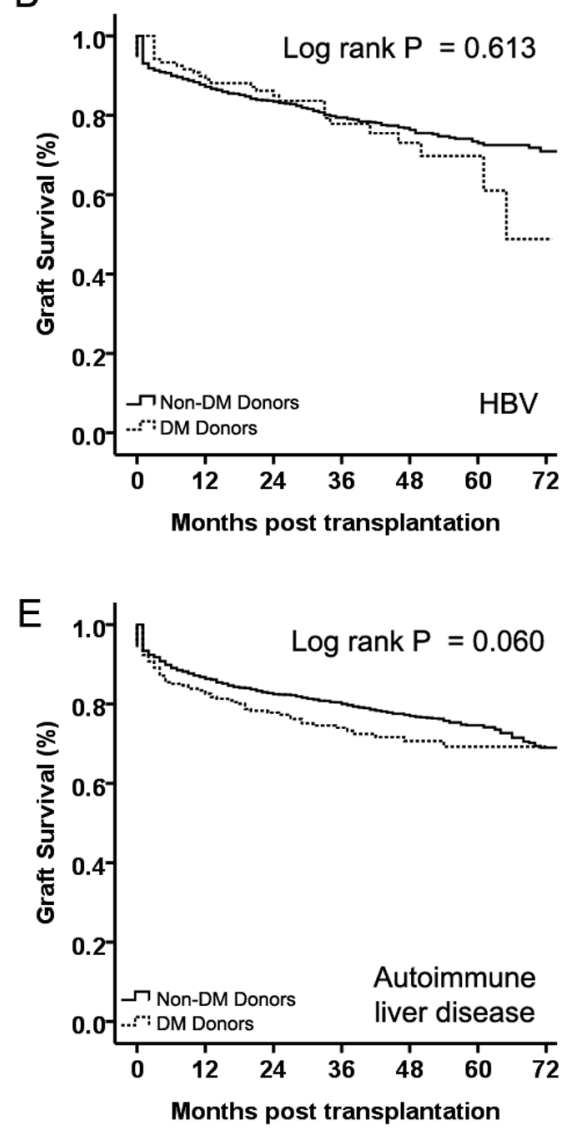

C

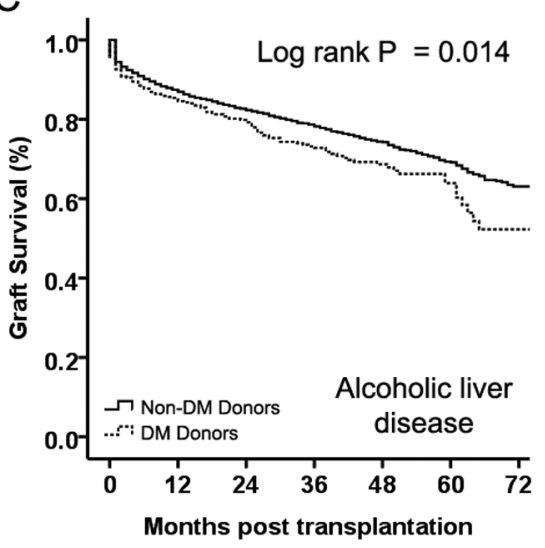

F

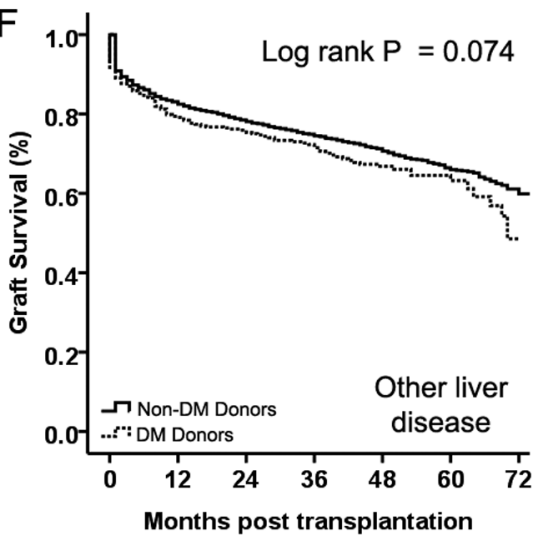

Figure 2. Kaplan-Meier survival curves comparing graft survival of recipients from diabetes mellitus (DM) and non-DM donors according to underlying liver disease. A) HCV, (B) HBV, (C) alcoholic liver disease, (D) NASH, (E) autoimmune liver disease, and (F) other liver disease. Graft survival was significantly lower in recipients from DM donors with HCV infection and alcoholic liver disease. Graft survival was similar between recipients from DM and non-DM donors with HBV infection, NASH, autoimmune liver disease and other liver disease. Continuous line depicts recipients from DM donors and dashed line depicts recipients from non-DM donors.

doi:10.1371/journal.pone.0098104.g002

LT from DM donors was very comparable with the risks associated with these other, well-accepted predictors of risk. Furthermore, by considering the interactions between these risks, a more precise understanding of the impact of receiving a liver graft from a DM donor can be achieved. Increases in the risk of graft loss were noted among recipients from DM donors with HCV at the time of LT, whereas those without HCV experienced greatly

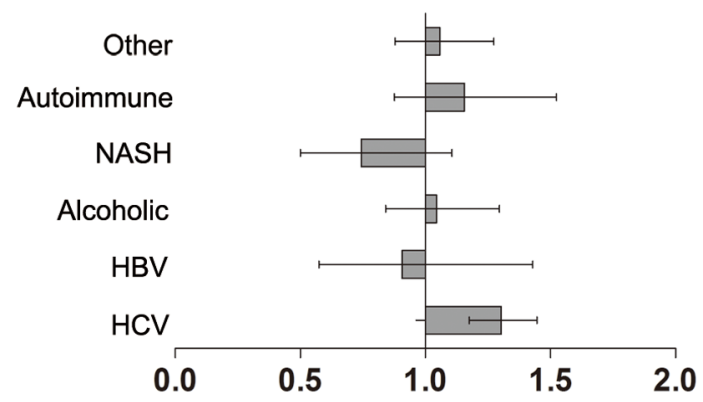

Figure 3. Hazard ratios (with $95 \% \mathrm{Cl}$ ) to compare the risk of graft loss after liver transplantation in recipients from diabetes mellitus (DM) donors with various types of underlying liver diseases.

doi:10.1371/journal.pone.0098104.g003 improved outcomes (even comparable with recipients from nonDM donors with the same underlying liver disease). In patients without HCV infection before LT, using DM donors was not independently associated with worse graft survival post-transplantation. Therefore, matching DM donors to recipients without HCV may be safe.

Several studies have noted that patients with a history of DM are associated with significant morbidity and mortality post-OLT $[3,6,11,12]$. However, few studies have addressed the impact on LT outcomes of receiving a liver graft from a donor with DM. Several studies have associated DM with hepatic steatosis, which is a benign form of non-alcoholic fatty liver disease (NAFLD) [13]. Increasing evidence suggests that NAFLD patients with DM are more likely to progress to NASH than NAFLD patients without DM $[14,15]$. NASH is a damaging form of NAFLD that leads to fibrosis and cirrhosis, resulting in poor graft function [16]. Studies have shown that grafts with moderate hepatic steatosis $(>30 \%)$ accelerate the progression of HCV-based disease, and should not be used for HCV patients with high MELD scores [17]. Another study found that moderate steatosis in combination with prolonged ischemic time resulted in worse transplantation outcomes in recipients with HCV [18]. The association of worse graft outcomes in HCV-positive recipients with liver grafts from donors with DM seen in the present study may be attributable to pre-existing graft 
steatosis and fibrosis induced by DM in the donor, which is further exacerbated by post-transplantation HCV recurrence with subsequent fibrosis.

The number of patients waiting for organ transplantation continues to grow, but donor organs remain in short supply. Over the past few years, steps have been taken to increase the number of organs available for OLT, including using split-LT and introducing living donor LT programs. Marginal donor grafts have also been explored thanks to improvements in surgical methods and immunosuppression. These marginal donors include those with steatotic livers, who are elderly, DCD donors, and donors infected with HCV [19,20]. Utilization of poorer-quality organs is in response to the increasing divergence between the supply and demand of organs. Improved understanding of the combined risks associated with different factors between donors and recipients is vital to decisions regarding utilization. Transplantation policy should incorporate adequate risk adjustment into measurement of the performance of transplantation centers and into improving informed consent to maximize the individual and societal benefits associated with transplantation.

Due to the registry-based nature of the present study, it had limitations that were mainly related to the data. The most important limitation was the inability to assess steatosis or fibrosis because donor biopsies were not carried out routinely. Secondly, any large database is subject to reporting bias, errors in data entry, and inaccuracies. The SRTR database is not immune to this problem, but these issues may be less of a concern in studies using the SRTR database because of the mandatory participation of all transplantation centers and the electronic editing system, which minimizes data-entry errors. Thirdly, the lack of information on previous and present use of anti-DM drugs as well as liver-biopsy details limited our ability to generalize the results.

The present study also had several extremely important strengths. We included the largest population of patients who have received DM liver grafts with the longest follow-up times available based on the SRTR database (which represents the entire transplantation population in the USA). The large sample size allowed more robust conclusions to be drawn in comparison

\section{References}

1. Danaei G, Finucane MM, Lu Y, Singh GM, Cowan MJ, et al. (2011) National, regional, and global trends in fasting plasma glucose and diabetes prevalence since 1980: systematic analysis of health examination surveys and epidemiological studies with 370 country-years and 2.7 million participants. Lancet 378: $31-$ 40 .

2. Whiting DR, Guariguata L, Weil C, Shaw J (2011) IDF diabetes atlas: global estimates of the prevalence of diabetes for 2011 and 2030. Diabetes Res Clin Pract 94: 311-321.

3. Samuelson AL, Lee M, Kamal A, Keeffe EB, Ahmed A (2010) Diabetes mellitus increases the risk of mortality following liver transplantation independent of MELD score. Dig Dis Sci 55: 2089-2094.

4. Kim WR, Stock PG, Smith JM, Heimbach JK, Skeans MA, et al. (2013) OPTN/SRTR 2011 Annual Data Report: liver. Am J Transplant 13 Suppl 1: 73-102.

5. Narayanan Menon KV, Nyberg SL, Harmsen WS, DeSouza NF, Rosen CB, et al. (2004) MELD and other factors associated with survival after liver transplantation. Am J Transplant 4: 819-825.

6. John PR, Thuluvath PJ (2001) Outcome of liver transplantation in patients with diabetes mellitus: a case-control study. Hepatology 34: 889-895.

7. Wahlstrom HE, Cooper J, Gores G, Rosen C, Wiesner R, et al. (1991) Survival after liver transplantation in diabetics. Transplant Proc 23: 1565-1566.

8. Nair S, Verma S, Thuluvath PJ (2002) Obesity and its effect on survival in patients undergoing orthotopic liver transplantation in the United States. Hepatology 35: 105-109.

9. Levine GN, McCullough KP, Rodgers AM, Dickinson DM, Ashby VB, et al. (2006) Analytical methods and database design: implications for transplant researchers, 2005. Am J Transplant 6: 1228-1242. with previous case reports with smaller sample sizes. Moreover, this is the first survival analysis study concerning post-transplantation graft survival of recipients who received liver grafts from subjects with DM. The SRTR database collects detailed pretransplantation variables that are known predictors of posttransplantation survival and adjusts for these variables to provide a less confounded assessment of the true effect of receiving a liver graft from a subject with DM on post-transplantation outcome. The limitations mentioned above affected our ability to confirm the reasons underlying our findings, but they did not affect the validity of our primary analyses of the survival of patients and grafts.

In summary, using the largest dataset available for analyses and the longest follow-up periods available to date, we showed that graft survival in recipients with grafts from subjects with DM were worse than those with non-DM grafts when other predictors of post-transplantation survival were taken into account. However, allocating these DM donors to patients without HCV could also result in similar post-transplantation outcomes with non-DM donors. With careful implementation and informed consent from the recipients, matching these DM donors to recipients without HCV may be safe.

\section{Acknowledgments}

The data reported here have been supplied by the Minneapolis Medical Research Foundation (MMRF) as the contractor for the Scientific Registry of Transplant Recipients (SRTR). The interpretation and reporting of these data are the responsibility of the authors and in no way should be seen as an official policy of or interpretation by the SRTR or the U.S. Government.

\section{Author Contributions}

Conceived and designed the experiments: J. Zheng CML WLW. Performed the experiments: JX J. Zhou ZWL. Analyzed the data: J. Zheng ZHH. Contributed reagents/materials/analysis tools: ZWL ZHH GML WLW. Wrote the paper: J. Zheng.

10. Feng S, Goodrich NP, Bragg-Gresham JL, Dykstra DM, Punch JD, et al. (2006) Characteristics associated with liver graft failure: the concept of a donor risk index. Am J Transplant 6: 783-790.

11. Blanco JJ, Herrero JI, Quiroga J, Sangro B, Gomez-Manero N, et al. (2001) Liver transplantation in cirrhotic patients with diabetes mellitus: midterm results, survival, and adverse events. Liver Transpl 7: 226-233.

12. Shields PL, Tang H, Neuberger JM, Gunson BK, McMaster P, et al. (1999) Poor outcome in patients with diabetes mellitus undergoing liver transplantation. Transplantation 68: 530-535.

13. Kotronen A, Juurinen L, Tiikkainen M, Vehkavaara S, Yki-Jarvinen H (2008) Increased liver fat, impaired insulin clearance, and hepatic and adipose tissue insulin resistance in type 2 diabetes. Gastroenterology 135: 122-130.

14. Hossain N, Afendy A, Stepanova M, Nader F, Srishord M, et al. (2009) Independent predictors of fibrosis in patients with nonalcoholic fatty liver disease. Clin Gastroenterol Hepatol 7: 1224-1229, 1229 e1221-1222.

15. Angulo P, Keach JC, Batts KP, Lindor KD (1999) Independent predictors of liver fibrosis in patients with nonalcoholic steatohepatitis. Hepatology 30: 13561362.

16. Farrell GC, Larter CZ (2006) Nonalcoholic fatty liver disease: from steatosis to cirrhosis. Hepatology 43: S99-S112.

17. Nocito A, El-Badry AM, Clavien PA (2006) When is steatosis too much for transplantation? J Hepatol 45: 494 499.

18. Briceno J, Ciria R, Pleguezuelo M, Naranjo A, Sanchez-Hidalgo J, et al. (2007) Contribution of marginal donors to liver transplantation for hepatitis $\mathrm{C}$ virus infection. Transplant Proc 39: 2297-2299.

19. Nadig SN, Bratton CF, Karp SJ (2007) Marginal donors in liver transplantation: expanding the donor pool. J Surg Educ 64: 46-50.

20. Busuttil RW, Tanaka K (2003) The utility of marginal donors in liver transplantation. Liver Transpl 9: 651-663. 